\title{
Segmentation of Focal Cortical Dysplasia Lesions Using a Feature-Based Level Set
}

\author{
O. Colliot, T. Mansi, N. Bernasconi, V. Naessens, \\ D. Klironomos, and A. Bernasconi
}

Montreal Neurological Institute, McGill University, Montreal, Canada

\begin{abstract}
Focal cortical dysplasia (FCD), a malformation of cortical development, is an important cause of medically intractable epilepsy. FCD lesions are difficult to distinguish from non-lesional cortex and their delineation on MRI is a challenging task. This paper presents a method to segment FCD lesions on T1-weighted MRI, based on a 3D deformable model, implemented using the level set framework. The deformable model is driven by three MRI features: cortical thickness, relative intensity and gradient. These features correspond to the visual characteristics of FCD and allow to differentiate lesions from normal tissues. The proposed method was tested on 18 patients with FCD and its performance was quantitatively evaluated by comparison with the manual tracings of two trained raters. The validation showed that the similarity between the level set segmentation and the manual labels is similar to the agreement between the two human raters. This new approach may become a useful tool for the presurgical evaluation of patients with intractable epilepsy.
\end{abstract}

\section{Introduction}

Malformations of cortical development (MCD) have been increasingly recognized as an important cause of medically intractable focal epilepsy. Focal cortical dysplasia (FCD) [1], a malformation due to abnormal neuroglial proliferation, is the most frequent MCD in patients with intractable extra-temporal epilepsy [2]. Epilepsy surgery, consisting in the removal of the FCD lesion, is an effective treatment for these patients. However, freedom from seizures after surgery is closely related to the resection of the whole lesion [3]. The precise delineation of lesions is thus important for surgical planning in epilepsy.

Magnetic resonance imaging (MRI) plays a pivotal role in the presurgical evaluation of patients with intractable epilepsy. Although MRI has allowed the recognition of FCD in an increased number of patients, standard radiological evaluation fails to identify lesions in a large number of cases due to their subtlety and the complexity of the cortex convolution [3. Moreover, the spatial extension of the lesions is difficult to define on the MRI. The segmentation of FCD is thus a challenging image analysis application as the lesions are often subtle, difficult to differentiate from the normal cortex, of variable size, position and shape, and with ill-defined boundaries. 
Recently, image analysis techniques have been developed to detect FCD lesions automatically on MRI, relying on different types of voxel-wise analysis [4, 5]. In particular, computational models of FCD characteristics [6] and a Bayesian classifier for lesion detection [4] were previously proposed by our group. While these approaches successfully identify the FCD in a majority of patients, they provide a very limited coverage of the lesion (about 20\%) and thus cannot be considered as segmentation techniques. Therefore, to our knowledge, the question of FCD segmentation has never been addressed.

This paper presents a method for segmenting focal cortical dysplasia (FCD) lesions on T1-weighted MRI, based on a level set deformable model driven by MR features of these lesions. The method partly relies on our aforementioned detection approaches [6, 4]. However, our target application is FCD segmentation and not detection. The computational models of FCD features are used to drive a level set deformable model and the FCD classifier is used only to obtain a starting point for the segmentation procedure.

\section{Methods}

Our approach relies on a 3D deformable model, based on the level set method. Driving the deformable model with image gray levels would be inadequate as this attribute is insufficient to distinguish the lesion from the normal cortex. Instead, we propose a model guided by a probability map derived from FCD features. These features correspond to the visual characteristics of FCD: cortical thickening, a blurred transition between gray matter (GM) and white matter (WM), and hyperintense signal within the dysplastic lesion [7.

Additionally, it is necessary to provide a starting point for the level set evolution. To this purpose, we used our previously developed FCD classifier [4], under supervision of an expert user.

\subsection{Probabilistic Modeling of FCD Features}

To quantitatively evaluate the visual MR characteristics of FCD, we made use of our previous computational models (more details can be found in [6]). A cortical thickness map, denoted as $T h$, is computed by solving Laplace's equation over the cortical ribbon. Hyperintense signal is represented using a relative intensity index defined as $R I(x)=1-\left|B_{g}-I(x)\right| / B_{g}$ where $I(x)$ is the intensity at voxel $x$ and $B_{g}$ is the boundary intensity between GM and WM. Blurring of the GM/WM transition is modeled with a gradient magnitude map, denoted as $G r$. These three characteristics define a vector-valued feature map $f(x)=(T h(x), R I(x), G r(x))$ at each point $x$ in the image space.

We then performed a supervised learning to estimate the probability of different tissue classes in the brain given the feature vector $f$. Four different classes, denoted as $c$, were considered: gray matter (GM), white matter (WM), cerebrospinal fluid (CSF) and the FCD lesion (L). Normal tissues were segmented using a histogram-based approach with automated threshold, while the FCD lesions were painted by trained observers (see Section 3 ). Conditional probabilities 
$P(f(x) \mid c)$ for each class $c$ were modeled using a trivariate normal distribution and estimated using the maximum likelihood on a learning set of patients. The posterior probabilities $P(c \mid f(x))$ were then obtained by Bayes' rule. As the size of FCD lesions is variable, we assumed equal prior probabilities for the different classes.

Figure 1 presents an example of the three feature maps and of the posterior probability maps in a patient with FCD.
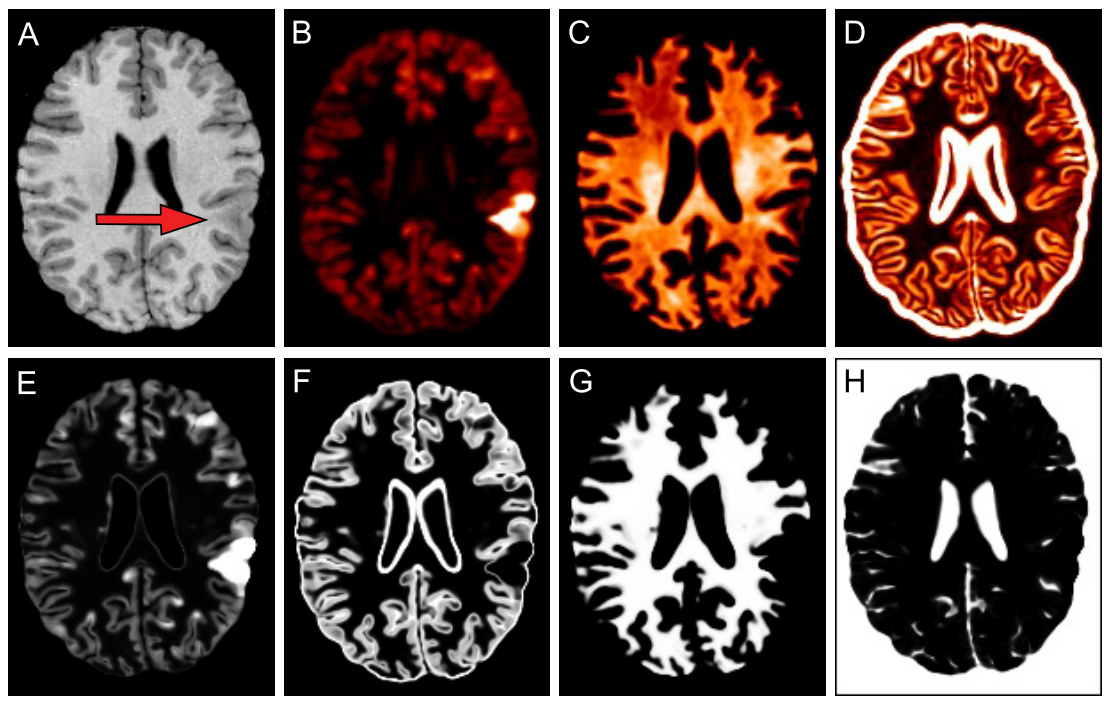

Fig. 1. Probabilistic modeling of FCD features. Upper panels: T1-weighted MRI where the FCD lesion is indicated by the arrow (A), cortical thickness map (B), relative intensity map (C), gradient map (D). The lesion is characterized by higher cortical thickness, higher relative intensity and lower gradient. Lower panels: probability maps of the lesion class (E), GM (F), WM (G) and CSF (H).

\subsection{Feature-Based Deformable Model}

Based on the previous features, the deformable model was designed to separate the lesion from the non-lesional regions. The region competition approach proposed by Zhu and Yuille [8] is well adapted to our purpose. It aims at segmenting an image into several regions by moving the interfaces between them. The evolution of the interfaces is driven by functions indicating the membership to each region. In our case, these functions can be derived from the FCD features.

We intended to isolate the FCD lesion from the non-lesional region, which is composed of three different classes (GM, WM, CSF). However, the boundaries between these three non-lesional classes were of no interest for our application. Thus, region competition occurred in each point between the lesion class and the most probable non-lesional class. The membership to the lesional region 
was defined as $R_{\mathrm{L}}(x)=P(\mathrm{~L} \mid f(x))$ which is the previously computed posterior probability of the lesion class. The non-lesional region was modeled by $R_{\mathrm{NL}}(x)=$ $\max \{P(\mathrm{GM} \mid f(x)), P(\mathrm{WM} \mid f(x)), P(\mathrm{CSF} \mid f(x))\}$.

The feature-based deformable model describes the evolution of the interface (or surface in 3D) $S$ of the lesional region, according to those membership functions and a regularization term. The motion of a point $u$ belonging to $S$ is defined as:

$$
\frac{\partial u}{\partial t}=\alpha\left[R_{\mathrm{NL}}(u)-R_{\mathrm{L}}(u)\right] \mathbf{n}_{u}+\epsilon \kappa_{u} \mathbf{n}_{u}
$$

where $\mathbf{n}_{u}$ is the inward normal to $S$ at point $u$ (directed towards the interior of the lesion), $\kappa_{u}$ is the mean curvature and $\alpha$ and $\epsilon$ are weighting coefficients.

In the previous equation, $\alpha\left[R_{\mathrm{NL}}(u)-R_{\mathrm{L}}(u)\right]$ is a feature-based term and $\epsilon \kappa_{u}$ is a regularity term producing a smooth surface. If $R_{\mathrm{L}}(u)>R_{\mathrm{NL}}(u)$, meaning that the most probable class for point $u$ is the lesion, the surface $S$ will be expanded, in order to include this point. On the contrary, if $R_{\mathrm{NL}}(u)>R_{\mathrm{L}}(u)$, meaning that this point should belong to one of the three non-lesional classes, the surface will be shrunk.

\subsection{Level Set Evolution}

The motion equation obtained for the feature-based deformable model was implemented using the level set method [9,10. The principle of this method is to define the surface $S$ as the zero level set of a higher dimensional function $\phi$, called the implicit function:

$$
\phi(S(t), t)=0
$$

As an implicit function $\phi$, we chose the classical signed distance to the surface $S$, with negative values in the interior of $S$. The evolution was then performed on the function $\phi$ and the embedded surface $S$ was deformed implicitly. Level set deformable models present several advantages over traditional ones: no parameterization of the surface is necessary, topology changes are handled naturally and the result is less sensitive to the initialization.

Using the derivation from curve motion to level set evolution [10, the featurebased deformable model can be described by:

$$
\left.\frac{\partial \phi}{\partial t}(u)=\alpha\left[R_{\mathrm{NL}}(u)\right)-R_{\mathrm{L}}(u)\right]|\nabla \phi(u)|+\epsilon \kappa_{u}|\nabla \phi(u)|
$$

The previous evolution equation can be seen as a particular case of the one proposed in [11].

This equation was implemented using the numerical scheme proposed in 10 chap.6]. To reduce the computational complexity, we made use of the narrowband method [12]. A sub-voxel reinitialization of the implicit function at fixed time steps was performed to maintain the distance function [13]. 


\section{Experiments and Results}

\subsection{Subjects and Image Preparation}

We selected 24 patients (13 males, mean age $\pm \mathrm{SD}=24 \pm 8$ ) with MRI-visible FCD. The Ethics Board of our Institution approved the study, and written informed consent was obtained from all participants.

3D MR images were acquired on a $1.5 \mathrm{~T}$ scanner using a T1-fast field echo sequence $(T R=18, T E=10,1$ acquisition average pulse sequence, flip angle= $30^{\circ}$, matrix size $=256 \times 256, \mathrm{FOV}=256$, thickness $\left.=1 \mathrm{~mm}\right)$ with an isotropic voxel size of $1 \mathrm{~mm}^{3}$. All images underwent automated correction for intensity non-uniformity and intensity standardization [14, automatic registration into stereotaxic space [15] and brain extraction [16]. Classification of brain tissue in GM, WM and CSF was done using an histogram-based method with automated threshold [6].

\subsection{Manual Segmentation}

Lesions were delineated independently on 3D MRI by two trained raters (VN and DK) using a software which allows painting in each of the three directions of the space. The corresponding manually labeled datasets are further denoted as $M_{1}$ and $M_{2}$. Interrater agreement was assessed using the similarity index $S=2 \frac{n\left\{M_{1} \cap M_{2}\right\}}{n\left\{M_{1}\right\}+n\left\{M_{2}\right\}}$ (where $n\{M\}$ is the number of elements in set $M$ ), which is a special case of kappa statistic since the vast majority of voxels are nonlesional [17.

\subsection{Results}

Initialization. The FCD classifier 4 is used to initialize the deformable model. It successfully identified the lesion in $18(18 / 24=75 \%)$ patients. We assessed the possibility of segmenting the six undetected lesions with a manual initialization of the procedure. However, the segmentation failed in these cases because their features where not sufficiently discriminant. The evaluation was thus done on the 18 detected lesions.

Manual Segmentation. For the 18 manual labels, the mean interrater similarity index was $0.62 \pm 0.19$ (range $=0.22$ to 0.84 ).

Level Set Segmentation. We compared the automated segmentations to the sets of manual labels using the similarity index $S$ presented above. The evaluation was performed using a leave-one-out approach: for the segmentation of a given patient, this patient was excluded from the learning set (Section 2.1). This approach avoids the introduction of bias in the result. All results were obtained with $\alpha=0.8$ and $\epsilon=0.2$ in Equation 3 Moreover, we computed the similarity obtained with the FCD classifier 4 to evaluate the added value of the level set. Results are reported in Table 1 Figures 2 and 3 present the segmentations obtained in two patients with FCD. 

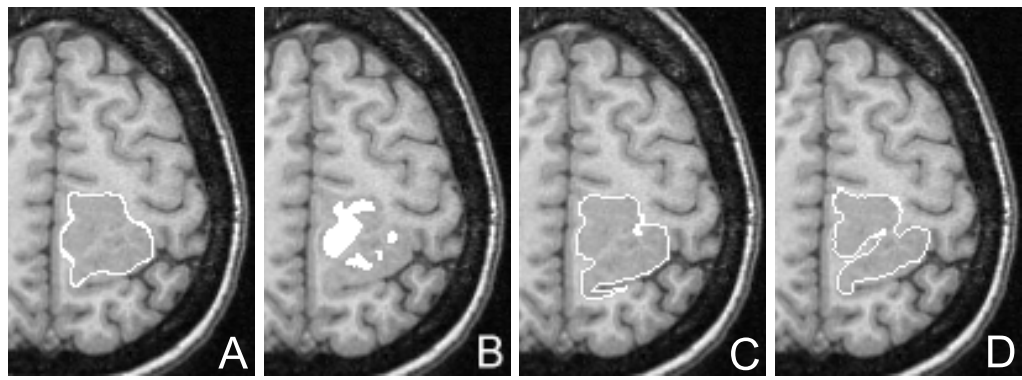

Fig. 2. Results of FCD segmentation: level set segmentation (A), initialization (B), manual tracing $M_{2}(\mathrm{C})$, manual tracing $M_{1}(\mathrm{D})$

Table 1. The table presents the similarity indices for the level set and the FCD classifier with respect to the two manual tracings, as well as the interrater similarity. Results are reported as mean $\pm \mathrm{SD}$ with the range in parentheses.

\begin{tabular}{|c|c|c|}
\hline & $M_{1}$ & $M_{2}$ \\
\hline Level set & $0.62 \pm 0.16(0.32$ to 0.84$)$ & $0.63 \pm 0.12(0.43$ to 0.79$)$ \\
Classifier & $0.30 \pm 0.17(0.11$ to 0.64$)$ & $0.31 \pm 0.17(0.07$ to 0.59$)$ \\
\hline \multicolumn{2}{|c|}{ Interrater $\left(M_{1}\right.$ vs. $\left.M_{2}\right)$} & $0.62 \pm 0.19(0.22$ to 0.84$)$ \\
\hline
\end{tabular}
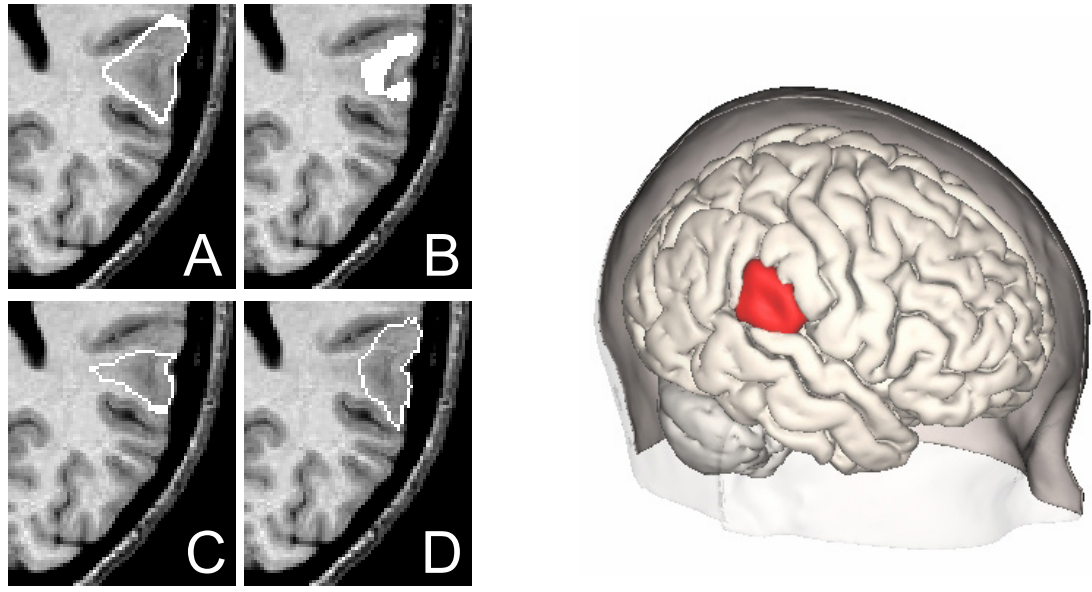

Fig. 3. Results of FCD segmentation. Left panels: level set segmentation (A), initialization (B), manual tracing $M_{2}(\mathrm{C})$, manual tracing $M_{1}(\mathrm{D})$. Right panel: 3D rendering of the FCD lesion segmentation together with the cortical surface. 
To assess the robustness of our method with respect to the choice of the learning dataset, the procedure was also evaluated using a learning on the labels $M_{2}$ (in Table 1 the results are obtained using the labels $M_{1}$ as a learning set). Similarity indices for the level set (LS) were: LS vs. $M_{1}=0.62 \pm 0.16$ (range $=0.33$ to 0.82 ) and LS vs. $M_{2}=0.62 \pm 0.11$ (range $=0.43$ to 0.77 ).

\section{Discussion}

In this study, we proposed and evaluated a method for segmenting FCD lesions on MRI. We introduced a feature-based level set, driven by known MR characteristics of FCD. Probability maps of these features corresponding to FCD and normal tissue classes were estimated in order to guide the level set evolution.

On MRI, FCD lesions possess ill-defined contours and are not easily differentiable from normal cortex. Moreover, there is no available gold standard for evaluating the delineation of these lesions. For this reason, we compared the level set segmentation to the manual tracings of two trained observers. The interrater similarity was 0.62 which corresponds to a substantial agreement, in particular when keeping in mind the difficulty of FCD segmentation. The level set segmentations achieved a degree of similarity of 0.63 and 0.62 with the two sets of manual labels, which again constitutes a good agreement.

The similarities achieved by the level set are also very close to the interrater agreement (both were computed on the 18 detected lesions). A significant portion of the remaining differences between automated and manual labels is probably due to the interrater variability rather than to the unability of the level set to recover the full extension of lesions. This can be seen in Figure 3 where the two raters decided to exclude different parts of the lesion (Panels C and D) while these parts were included in the automated segmentation (Panel A).

To our knowledge, there is no other published work on FCD segmentation that could be used for comparison to our results. Nevertheless, compared to the FCD classifier, our method achieved a similarity twice as large and therefore constitutes a significant improvement. However, it should be noted that this classifier was designed for FCD detection and not segmentation.

The results of the automated segmentation did not depend on the manual tracings (by one rater or the other) used for the learning step. This shows the robustness of our method with respect to the learning dataset. Moreover, no fine-tuning of the level set parameters was necessary and the same parameters were used for all patients. Furthermore, the method is fast thanks to the use of the narrow-band and the subvoxel reinitialization approaches (the mean computation time for the level set evolution was 4 minutes on a $\mathrm{PC} 1.6 \mathrm{GHz}$ ).

In conclusion, this paper demonstrates the effectiveness of a feature-based level set approach for the segmentation of FCD lesions. We do not advocate that the level set segmentation should be used in place of manual delineation but rather that it is a complementary tool. It has the potential to reduce user subjectivity and, more importantly, to unveil lesional areas that could be overlooked by visual inspection. This new method may become a useful tool for sur- 
gical planning in epilepsy. Future work includes further validation using other metrics, comparison with other image segmentation techniques and a detailed study of cases that were not detected by the classifier.

\section{References}

1. Taylor, D., Falconer, M., Bruton, C., Corsellis, J.: Focal dysplasia of the cerebral cortex in epilepsy. J Neurol Neurosurg Psychiatry 34 (1971) 369-387

2. Sisodiya, S.: Surgery for malformations of cortical development causing epilepsy. Brain 123 (2000) 1075-1091

3. Tassi, L., Colombo, N., et al.: Focal cortical dysplasia: neuropathological subtypes, EEG, neuroimaging and surgical outcome. Brain 125 (2002) 1719-1732

4. Antel, S., Collins, D., et al.: Automated detection of focal cortical dysplasia lesions using computational models of their MRI characteristics and texture analysis. NeuroImage 19 (2003) 1748-59

5. Wilke, M., Kassubek, J., Ziyeh, S., Schulze-Bonhage, A., Huppertz, H.: Automated detection of gray matter malformations using optimized voxel-based morphometry: a systematic approach. NeuroImage 20 (2003) 330-343

6. Antel, S., Bernasconi, A., et al.: Computational models of MRI characteristics of focal cortical dysplasia improve lesion detection. NeuroImage 17 (2002) 1755-60

7. Barkovich, A., Kuzniecky, R.: Neuroimaging of focal malformations of cortical development. J Clin Neurophysiol 13 (1996) 481-494

8. Zhu, S., Yuille, A.: Region competition: Unifying snakes, region growing, and bayes/MDL for multiband image segmentation. IEEE TPAMI 18 (1996) 884-900

9. Osher, S., Sethian, J.: Fronts propagating with curvature-dependent speed : algorithms based on Hamilton-Jacobi formulation. J Comp Phys 79 (1988) 12-49

10. Sethian, J.: Level-set methods and fast marching methods. 2nd edn. Cambridge University Press (1999)

11. Paragios, N., Deriche, R.: Geodesic active regions and level set methods for supervised texture segmentation. Int J Comp Vis 46 (2002) 223-247

12. Adalsteinsson, D., Sethian, J.: A fast level set method for propagating interfaces. J Comp Phys (1995) 269-277

13. Krissian, K., Westin, C.F.: Fast sub-voxel re-initialization of the distance map for level set methods. Patt Recog Letters (2005) In Press.

14. Sled, J., Zijdenbos, A., Evans, A.: A nonparametric method for automatic correction of intensity nonuniformity in MRI data. IEEE TMI 17 (1998) 87-97

15. Collins, D., et al.: Automatic 3D intersubject registration of MR volumetric data in standardized Talairach space. J Comput Assist Tomogr 18 (1994) 192-205

16. Smith, S.: Fast robust automated brain extraction. Hum Brain Mapp 17 (2002) $143-155$

17. Zijdenbos, A., Dawant, B., et al.: Morphometric analysis of white matter lesions in MR images: method and validation. IEEE TMI 13 (1994) 716-724 